Topiques, études satoriennes

Topoï Studies, Journal of the SATOR

\title{
Potentialités narratives de la nourriture : les choix d'Isabelle de Charrière
}

Suzan van Dijk

Volume 5, 2021

Le manger et le boire dans la fiction narrative

URI: https://id.erudit.org/iderudit/1081528ar

DOI: https://doi.org/10.7202/1081528ar

See table of contents

Publisher(s)

SATOR, Société d'Analyse de la Topique Romanesque d'Ancien Régime

ISSN

2369-4831 (digital)

Explore this journal

Cite this article

van Dijk, S. (2021). Potentialités narratives de la nourriture : les choix d'Isabelle de Charrière. Topiques, études satoriennes / Topoï Studies, Journal of the SATOR, 5, 143-160. https://doi.org/10.7202/1081528ar
Article abstract

In Isabelle de Charrière's novels, there are repeated scenes of meals where the protagonists talk about eating. The novelist uses them to characterize her characters and to create situations, which allow her to illustrate her theses. The confrontation between the correspondence - currently being digitized - and the novels clearly shows this link between her democratic convictions (expressed in some of the letters) and their staging, aiming to reach a wider audience.
This document is protected by copyright law. Use of the services of Érudit (including reproduction) is subject to its terms and conditions, which can be viewed online.

https://apropos.erudit.org/en/users/policy-on-use/ 


\section{Potentialités narratives de la nourriture : les choix d'Isabelle de Charrière}

Depuis plusieurs années la numérisation de la correspondance d'Isabelle de Charrière est en cours de préparation. Nous en avons fait un projet qui ne dépend pas complètement de la machine, et auquel collaborent des bénévoles : intéressé-e-s justement par le contenu des lettres ${ }^{1}$. Le travail sur ces lettres nous a permis de nous rendre compte, une fois de plus, combien il y a de liens étroits reliant entre elles les fictions (narratives et théâtrales) et la correspondance de cette écrivaine hollando-suisse de la fin du XVIII ${ }^{\mathrm{e}}$ siècle : les lettres peuvent être lues comme un métadiscours sur les romans et les pièces ${ }^{2}$.

Les relectures qu'impliquait la numérisation m'amènent à considérer ici la topique liée à la nourriture dans les deux corpus : telle que repérable dans les lettres, et comme fonctionnant dans les romans d'Isabelle de Charrière. Les rôles joués dans sa propre vie par les repas, les aliments, leur préparation, leur digestion sont évidemment familiers à la romancière. Ils pourraient se retrouver dans ses fictions, où elle aurait pu leur donner une signification supplémentaire. La possibilité - grâce au numérique - de faire des sondages rapides dans ce corpus d'environ 2600 lettres nous assure, pour ensuite approcher les romans, un point de départ qui n'est donc pas complètement arbitraire.

\section{Romancière et épistolière}

\footnotetext{
${ }^{1}$ Voir par exemple Suzan van Dijk et Maria Schouten, « Numériser les lettres de Belle de Zuylen : Un regard plus précis sur les rapports familiaux »(2013). Le travail préparatoire à cette édition, avec modernisation de l'orthographe et de la ponctuation et rajout de scans représentant le manuscrit, est réalisé dans le cadre de l'Institut Huygens pour l'Histoire des Pays-Bas (Amsterdam) grâce à la collaboration de quelques membres de l'Association Isabelle de Charrière. L'édition des $O C$ (voir n. 4) reste la source de référence : notamment la numérotation des lettres est maintenue, les lettres retrouvées depuis 1984 étant intercalées dans l'ordre chronologique avec une numérotation adaptée (voir par exemple la lettre mentionnée n. 23). En octobre 2019 ont d'abord été présentées les lettres (au nombre de 199) dont les manuscrits originaux se trouvent dans des archives aux Pays-Bas. Elles sont accessibles à l'adresse : https://charriere.huygens.knaw.nl/. Il est prévu de présenter dans l'année à venir les 2400 lettres qui restent.

${ }^{2}$ Monique Moser-Verrey insiste également sur les liens entre les deux corpus, en présentant les lettres comme un « laboratoire d'écriture » qui fonctionnerait comme une « archive » préparatoire aux romans (voir son Isabelle de Charrière, salonnière virtuelle : Un itinéraire d'écriture au XVIIIe siècle, 2013, p. 124).
} 
Malgré l'intérêt qui allait être reconnu à son œuvre fictionnelle (romans brefs et pièces

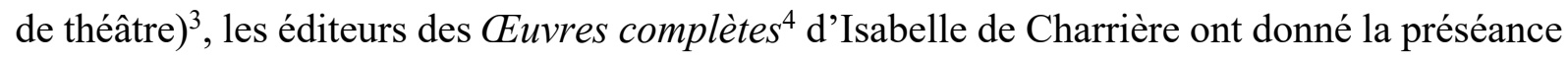
à ses lettres (t. I-VI); elles sont suivies par les ouvrages écrits à l'intention d'un public : théâtre (t. VII), romans (t. VIII-IX), et (t. X) vers, essais et compositions musicales. A première vue ce classement peut sembler correspondre à une plus grande importance accordée à la personne de l'auteure qu'à son $\varkappa^{2} v e^{5}$. On peut se demander ce que Charrière en aurait pensé, elle qui insistait régulièrement sur son désir de communiquer ses idées à un lectorat ${ }^{6}$, et qui demandait à ses correspondants qu'ayant lu ses lettres, ils les brûlent ${ }^{7}$.

Mais il est sans doute vrai qu'elle était une femme extraordinaire. Née dans la vieille aristocratie hollandaise au Château de Zuylen près d'Utrecht, Isabella van Tuyll van Serooskerken (1740-1805), couramment appelée Belle de Zuylen, prenait jusqu'à un certain point ses distances par rapport à son milieu d'origine. Pendant les années dominées - pour les parents - par le besoin de lui trouver un mari qui convienne (entre 1762 et 1770), elle entretenait une correspondance confidentielle avec le colonel suisse - marié - David-Louis Constant d'Hermenches installé à La Haye. Puis elle décida de se marier hors de sa propre classe. Le mariage eut lieu en 1771, et avec son mari Charles-Emmanuel de Charrière elle quitta sa patrie pour la Suisse francophone, où elle s'inséra facilement : elle connaissait un peu le pays, grâce notamment à la gouvernante suisse qu'elle avait eue étant enfant. La langue ne posait donc pas de problème, au contraire.

La plupart de ses publications, ainsi que le plus grand nombre de ses lettres ${ }^{8}$, datent de la «période suisse» de sa vie. Par contre la «période hollandaise», avec une supposée « rébellion » contre son milieu d'origine, semble attirer davantage le public moderne - pas

\footnotetext{
${ }^{3}$ Deux historiens néerlandais écrivent sur ses romans qu'ils « displayed a subtle psychological sensitivity, that would not appear in Dutch novels for many years »; « faisaient preuve d'une sensibilité psychologique subtile qui ne devait pas apparaître dans les romans hollandais avant de nombreuses années ", notre traduction, (Joost Kloek et Wijnand Mijnhardt, Dutch Culture in a European Perspective 2: 1800; Blueprints for a National Community, 2004, p. 430), et selon Yves Citton « elle composa une série de brefs chefs-d'œuvre consacrés à l'exploration des multiples façons dont nos sentiments intérieurs sont inextricablement liées à nos relations avec le monde extérieur » ( dans « Les spectres de la multiplicité. La littérature du XVIIIe siècle revisitée depuis ses dehors », 2014, en ligne : https://hal.archives-ouvertes.fr/hal-01373195/document [cons. 2-3-2021]). Il faut préciser que c'est sans doute la présence de ces œuvres dans les Euvres complètes qui est à l'origine de leur renommée actuelle. ${ }^{4}$ Euvres complètes d'Isabelle de Charrière/Belle de Zuylen. 1979-1984, dorénavant indiqué par OC.

${ }^{5}$ Les éditeurs eux-mêmes renvoient, à propos de ces lettres, à la réputation qu'elles avaient acquise grâce à des prédécesseurs comme Sainte-Beuve et Philippe Godet, premier biographe de Charrière. (OC, t.I, p. 7) ${ }^{6}$ Yvette Went-Daoust a signalé dès 1994 que « Mme de Charrière est moins romancière qu'essayiste », l'intrigue de ses romans « ne sert principalement qu'à illustrer des thèses » dans Went-Daoust, « La place des Lettres neuchâteloises dans le roman épistolaire du XVIII' siècle », 1994, p. 194.

${ }^{7}$ Et en effet bon nombre de lettres ont disparu ; voir Suzan van Dijk et Maria Schouten, « La correspondance d'Isabelle de Charrière (1740-1805). Survie et destruction des lettres comme thématiques de l'épistolière », 2019. ${ }^{8}$ C'est-à-dire : de celles qui ont survécu. Nous disposons de 422 lettres datant des années entre 1753 et 1771, et de 2218 pour la période 1771-1805. Grâce à la numérisation il sera plus facile d'évaluer le nombre des lettres qui ont disparu (voir note précédente).
} 
uniquement aux Pays-Bas d'ailleurs ${ }^{9}$. En France la correspondance avec Constant d'Hermenches a été publiée séparément sous le titre Une liaison dangereuse ${ }^{10}$ - tendant à convertir en personnage de roman celle que l'on connaît comme auteure...

$\mathrm{Au} v \mathrm{vu}$ des réactions des lecteurs et lectrices actuel-le-s, les éditeurs des $O C$ n'ont probablement pas eu tort de mettre en avant les documents privés : il semble bien que le ton très personnel que Belle de Zuylen/Isabelle de Charrière adopte dans ses lettres, adressées souvent à des personnalités intéressantes qui étaient des ami-e-s intimes, permet un contact direct aussi entre elle et son public d'aujourd'hui ${ }^{11}$. L'intérêt de la part également d'un public non-professionnel contribue à justifier notre édition en ligne, qui sera plus facile d'accès que celle, diplomatique, contenue dans les Euvres complètes. A terme, elle inclura également des traductions, notamment en néerlandais ${ }^{12}$.

Dans le cadre de ce volume nous nous concentrons donc sur le phénomène « profondément humain » qu'est le besoin de manger. Comme on peut le constater, il occupe quelque place et a une certaine importance, aussi bien dans les lettres que dans les romans de Belle de Zuylen/Isabelle de Charrière. Ce n'est pas que la nourriture - comme produit et comme activité - représente une préoccupation majeure : la romancière ne semble pas avoir été ellemême une grande mangeuse, encore moins une bonne cuisinière ${ }^{13}$. A Zuylen, chez les Tuyll, on était sobre, et à Colombier Isabelle suit l'exemple parental : «On mange ici assez frugalement et j'y tiens la main », écrit-elle à sa belle-sœur Dorothea van Tuyll en $1799^{14}$. Ces remarques mêmes sur la frugalité sont pertinentes par rapport au rôle que joue la nourriture dans sa vie, et donnent une première impression de son attitude en la matière.

Mais la véritable question à traiter est celle qui consiste à voir si, en tant que romancière, Isabelle de Charrière a mis en œuvre des « nourritures fictionnelles », que ce soit pour caractériser des personnages, ou pour amener des situations qui lui sont significatives et

\footnotetext{
${ }^{9}$ Aux Pays-Bas, on aime citer - dans la traduction de Greetje van den Bergh - une phrase provenant d'une lettre (98, datée 18-19 juin 1764, adressée à James Boswell, étudiant alors à Utrecht): «Je n'ai pas les talents subalternes » («Ik heb geen talent voor ondergeschiktheid »).

${ }^{10}$ Isabelle Vissière et Jean-Louis Vissière (éds.), Isabelle de Charrière. Une liaison dangereuse : Correspondance d'Isabelle de Charrière avec Constant d'Hermenches, 1760-1776, 1991.

${ }^{11}$ L'écrivaine néerlandaise Nelleke Noordervliet est très explicite à ce propos. Elle déclare ressentir comme une intimité entre Charrière épistolière et elle-même, alors que pour elle ses romans seraient restés « trop empreints des conventions romanesques du XVIII ${ }^{\mathrm{e}}$ siècle ». Voir Nelleke Noordervliet, Volg je eigen weg (Suivre son propre chemin), 2013, p. 47-48.

${ }^{12}$ Celles qui ont déjà été publiées par Greetje van den Bergh (voir la bibliographie) et d'autres, qui pourraient être préparées par des étudiants dans le cadre de leurs études (une collaboration est en train de se créer avec le département de français de l'Université de Nimègue).

${ }^{13}$ Dans ses lettres, elle n'en parle guère; à Colombier c'étaient ses belles-sœurs qui étaient responsables du ménage. L'idée qu'il pourrait y avoir un lien privilégié entre la féminité de l'auteure et les préoccupations nourricières ne s'applique probablement pas à Isabelle de Charrière, ni aux femmes de sa classe.

${ }^{14}$ Lettre 2059 à sa belle-sœur Dorothea de Tuyll-de Pagniet, 16 ou 17 juillet 1799, OC, t. V, p. 600.
} 
importantes. Et surtout, les topoi potentiels dont on trouverait des occurrences, seraient-ils (1) exclusivement liés à la nourriture, et (2) caractéristiques de notre auteure ?

\section{Manger et boire, d'après la correspondance privée}

Voyons d'abord, comme point de départ, la correspondance et le vécu de la romancière : quel est le rôle qu'a dû jouer - malgré la frugalité ambiante - le « manger et boire » pour Belle de Zuylen / Isabelle de Charrière dans la vie de tous les jours, telle qu'elle se reflète dans les lettres? Les travaux liés à la numérisation ont permis de vérifier la présence et le co-texte d'un certain nombre de mots-clés renvoyant au « boire et manger ».

Des termes - mots-clés potentiels - comme repas, manger, souper, à table, café, pain, légumes ${ }^{15}$ se laissent retrouver dans des passages qui ressortent de deux catégories différentes. Il a été jugé utile, dans un premier temps, de ne pas privilégier ici l'une des deux, puisqu'elles sembleraient se compléter : repas, manger, souper, à table, etc. renvoient souvent à des moments plus ou moins fixes dans la journée, et signifient en fait «l'heure de manger ou de souper ", correspondant donc à un apparent besoin de structurer la journée. En revanche, des mots indiquant des boissons, mets ou victuailles précis (café, pain, légumes) mènent plutôt à des observations sur leurs mérites face à des ennuis de santé ou des problèmes de concentration, que ce soient les siens propres ou ceux dont des correspondants se seraient plaints dans leurs lettres.

La structure des journées, créée ou à créer grâce aux repas, tient à ce que non seulement on mange à des heures plus ou moins fixes, mais qu'aussi les repas sont pris (normalement) en commun. Ils présupposent, dans son milieu d'origine, une certaine activité du personnel : on ne pouvait improviser un repas. C'est donc par définition un moment où se passe une interaction entre les membres de la famille, des visiteurs ou des domestiques. Cette structuration, reconnaissable pour tous, crée des « attaches » permettant à l'épistolière de rendre compte, dans une lettre, de ce qui avait pu se passer dans une journée, en particulier au niveau des contacts interhumains :

Je vis l'autre jour M. Bicker; il parla fort de vous. C'était à Amerongen où il vint déjeuner en passant avec sa femme, une cousine et Perponcher cadet. Ils allaient à Clèves, je leur ai fort conseillé d'aller jusqu'à Dusseldorp ${ }^{16}$.

\footnotetext{
${ }^{15}$ Nombres d'occurrences respectives dans des lettres envoyées par Charrière elle-même : souper 97, manger 51, pain 26, café 24 , à table 23 , repas 13 , légumes 7.

${ }^{16}$ Lettre 370 à son frère Ditie, 9 juillet 1770, OC, t. II, p. 199.
} 
La qualité du déjeuner, les talents du cuisinier et la faim qui pourrait s'être manifestée n'entrent guère en ligne de compte ${ }^{17}$. Cela est encore moins le cas dans les recommandations où Isabelle de Charrière se donne quelque peine pour faire travailler Willem-René van Tuyll, le jeune neveu dont elle surveille à distance l'apprentissage du français. En tant que mentor elle exige de lui un emploi de temps structuré, auquel la régularité des repas est censée être utile : «Ecrivez tous les matins avant déjeuner une conjugaison tout entière, deux déclinaisons, à commencer par les pronoms, et vingt mots, à commencer par les adverbes. Ne déjeunez jamais que cela ne soit fait ${ }^{18} \gg$.

Quant à la nourriture en tant que telle, il en est surtout question pour ses capacités de prévenir différents types de maux, ou au contraire lorsqu'elle a occasionné des maladies, qu'il faudrait chercher à guérir par des herbes ou des plantes appropriées. En 1770, par exemple, Charrière décrit ainsi son propre état physique :

Mes nerfs sont fort dérangés : depuis plusieurs jours jusqu'à hier je n'ai pu manger la moindre chose sans avoir un quart d'heure après des douleurs affreuses dans les dents et l'oreille. [....]. Hier au soir je m'avisai de demander des anchois, je les attendis avec une impatience qu'on ne peut décrire, je les dévorai et je n'eus point les douleurs accoutumées, j'en ai fait mon déjeuner et mon dîner aujourd'hui, et je continue à être exempte de douleurs, mais toute ma personne est fatiguée ${ }^{19}$.

Il s'agit donc ici d'une «auto-médication », mais elle prodigue aussi volontiers des conseils alimentaires, parfois surprenants, à ses proches et connaissances, comme il ressort de l'exemple suivant, tiré d'une lettre à son amie Caroline de Sandoz :

Ma belle ${ }^{20}$, soignez-vous, je vous en prie. Je voudrais qu'à vos repas vous bussiez de la bière, faisant tiédir le verre dans la cavette du poêle. Si par hasard vous détestiez la bière, faites bouillir de l'eau et jetez-y un morceau de pain grillé et un peu d'écorce de citron. Cela s'appelle de l'eau panée et cela est fort bon. Essayez aussi le matin à jeun d'avaler cinq ou six morceaux de glace, gros chacun comme une noisette. Rien n'est plus fortifiant et on a guéri avec cela des dévoiements et des coliques et toute débilité. Vous déjeunerez bientôt après et vous vous trouverez avoir fort bon appétit. Si cela ne réussit pas, vous aurez recours à la rhubarbe ${ }^{21}$.

\footnotetext{
${ }^{17}$ Un mot comme « délicieux » apparaît 22 fois, dont deux fois dans des lettres signées Isabelle de Charrière. Il s'appliquerait plutôt à des « moments », des « jardins », des « plaisirs ».

${ }^{18}$ Lettre 1794 à son neveu Willem-René van Tuyll, 30-31 mai 1797, OC, t. V, p. 313.

${ }^{19}$ Lettre 377 à son cousin Frederik Christiaan van Reede et Athlone, 3 septembre 1770, OC, t. II, p. 211.

${ }^{20}$ Nous ne disposons pas de la lettre où Caroline de Sandoz a dû décrire ses maux.

${ }^{21}$ Lettre 1876 à Caroline de Sandoz-Rollin, le 8 janvier 1798, OC, t. V, p. 396.
} 
Le ton relativement pressant, voire impératif, qu'elle adopte est typique d'Isabelle de Charrière mentor, conseillère et «teaching devil », comme elle se dénommait dans une lettre à son neveu Willem-René22.

A côté des pouvoirs bénéfiques sur la santé, un autre aspect de la nourriture apparaît, qui peut mener à la décision, pour des raisons de principe, de ne pas manger tel ou tel mets : le refus d'un aliment représente alors une prise de position par rapport à une norme sociale plus ou moins généralement acceptée. Dans une lettre à un autre jeune neveu, frère du premier, elle se déclare non seulement sobre, mais - autant que possible - végétarienne : « Je crois que si je vivais seule, je ne mangerais ni viande ni bouillon. Déjà j'en mange fort peu, [...] quand je mangerais un poulet ou un pigeon, je croirais manger mon prochain ${ }^{23} »$. En effet, dans ses lettres, depuis son plus jeune âge, on voit Belle s'opposer activement à ce passe-temps « nobiliaire » qu'était la chasse. C'est ce qui lui créait des conflits avec deux de ses frères ${ }^{24}$, et qui allait donc jusqu'à influencer plus tard son régime alimentaire.

On constate que ces trois éléments - le besoin de structurer le temps, les raisons médicales ou diététiques, et les principes de vie - donnent lieu à des situations de conseil : en l'occurrence $^{25}$ c'est Isabelle de Charrière qui montre à sa «pupille » comment structurer sa journée, qui se connaît (du moins prétend se connaître) en herboristerie, et semble donner ses propres principes en exemple aux autres. Par le biais du discours épistolaire autour de la nourriture elle manifeste son instinct de guide et de mentor, et s'arroge une certaine autorité. Autrement dit, l'emploi de la nourriture ou des repas serait chez elle lié à celui du topos, ici épistolaire, du MENTORAT. Qu'en est-il dans ses romans?

\section{Manger et boire dans les romans}

\footnotetext{
${ }^{22}$ Lettre 1794 à son neveu Willem-René de Tuyll, 30-31 mai 1797, OC, t. V, p. 313. Voir à ce propos Madeleine van Strien-Chardonneau, «Isabelle de Charrière, pédagogue », 2006. Par rapport à ce Willem-René, le succès de ses efforts était d'ailleurs tout à fait relatif, comme le montre Paul Pelckmans dans « Adieu Poes que je chéris, quoiqu'il me mette de mauvaise humeur.... Isabelle de Charrière et Willem-René van Tuyll », 2006.

${ }^{23}$ Lettre 1966X05 (cf. n.1) à son neveu Charles-Louis de Tuyll, 23 novembre 1798, retrouvée récemment, voir Hein H. Jongbloed et Suzan van Dijk, « De vier 'nieuwe' brieven van Belle van Zuylen », 2012, p. 91-99.

${ }^{24}$ En 1769 , après la mort de sa mère, elle écrit à Ditie, son frère préféré : « Nulle société entre mes frères et moi que celle que la nécessité nous impose. Vincent est civil, froid et systématique, Guillaume inégal, souvent dur et impoli. [...] ils n'aiment que leur liberté et la chasse » (Lettre 345 à son frère Ditie, le 27 novembre 1769, OC, t.II, p. 156).

${ }^{25}$ Pour l'instant je n'ai pas vérifié les tendances qu'ont pu avoir les correspondants à envoyer de semblables conseils à Belle/Isabelle.
} 
Les narrations les plus importantes d'Isabelle de Charrière étant également accessibles en $\operatorname{ligne}^{26}$, on peut procéder à une comparaison assez précise. Dans les documents numérisés j'ai repéré les mêmes mots-clés, afin d'illustrer maintenant l'emploi qu'en tant que romancière elle fait de la nourriture par rapport aux personnages qu'elle crée, et des repas comme moyen de structurer son matériau narratif. Je prends comme exemples les ouvrages suivants : Le Noble (1763), Lettres neuchâteloises (1784), Lettres de Mistriss Henley (1784), Trois femmes (1795) et Sainte Anne (1799) - à savoir : son premier «conte moral » écrit à la $3^{\mathrm{e}}$ personne, deux romans épistolaires, et deux romans à la $3^{\mathrm{e}}$ personne avec comme narrateur le personnage de « l'Abbé de La Tour» (mais où l'épistolarité joue aussi un rôle important).

Comme l'a souligné à juste titre Monique Moser-Verrey, Isabelle de Charrière « consacre son œuvre romanesque et son théâtre à l'étude des relations interpersonnelles, [et] aux rituels d'interactions qui les caractérisent ${ }^{27} \gg$. Ces interactions - dont les repas seraient emblématiques - se passent entre personnages qui, appartenant assez souvent à des classes sociales différentes, arrivent à se côtoyer soit de façon «naturelle», soit par des hasards qu'aurait organisés la romancière: elle nous présente des nobles et des moins nobles, des seigneurs et des domestiques, des gens riches et d'autres qui le sont moins. Le nœud de l'intrigue est souvent un mariage à réaliser - situation où il est à la fois important et délicat de tenir compte des situations, des caractères, des habitudes des un-e-s et des autres.

Lorsque dans sa correspondance Isabelle de Charrière discute ses propres romans, leur écriture et leur réception, elle commente aussi cette grande diversité parmi les membres de son « personnel romanesque ». Elle explique alors qu'il s'agit là d'un projet, et qu'elle n'est pas forcément en train de présenter des réalités réelles ou des situations vraisemblables : «Partout dans ce que je ferai, je mettrai en scène des gens de la classe basse, pour leur faire jouer un rôle honnête, noble même à un certain point. C'est ma démocratie à moi. [...] l'on sera auprès de moi prince impunément, ainsi que laquais ${ }^{28} \gg$. Comme elle l'écrit à une amie qui vivait à la cour du Roi de Prusse : «[...] il n'y a pour moi ni grand seigneur que je respecte parce qu'il est grand seigneur, ni polisson que je dédaigne parce qu'il est un polisson ${ }^{29} »$.

\footnotetext{
${ }^{26}$ Le site de l'Association Isabelle de Charrière (http://www.belle-van-Zuylen.eu/site-charriere/fra/oeuvres.htm) et Athéna (http://athena.unige.ch/athena/) présentent une partie de l'œuvre romanesque. Les pièces de théâtre se trouvent seulement dans les $O C$, t. VII.

${ }^{27}$ Monique Moser-Verrey, Isabelle de Charrière, salonnière virtuelle : Un itinéraire d'écriture au XVIII siècle, 2013 , p. 70.

${ }^{28}$ Lettre 1359 à Ludwig Ferdinand Huber, 20 juin 1794, OC, t. IV, p. 466. Ici et dans la suite, les italiques dans les citations sont toujours les miennes.

${ }^{29}$ Lettre 811 à Henriette L'Hardy, 7-8 mai 1792, OC, t. III, p. 359. Voir aussi Suzan van Dijk, « Belle van Zuylen : schrijfster van adel, over de adel. Haar correspondentie digitaal beschikbaar », à paraître 2021. Je souligne.
} 
Pour illustrer cette égalité fondamentale qu'Isabelle de Charrière postule entre individus, il y avait une certaine logique à ce qu'elle se serve de ce qui est lié à notre état physique, comme par exemple le besoin de manger et la nourriture. Pour vérifier ce qui - dans les cinq romans sélectionnés - se passe à ce niveau, nous allons suivre l'ordre chronologique de leur parution.

\section{Le Noble (1763)}

Isabelle de Charrière accorde à la nourriture un rôle dès son premier roman. On y retrouve l'aversion de l'auteure contre la chasse et contre ceux qui mangent les « produits » de la chasse. Sur le baron d'Arnonville, père du personnage central Julie, on peut lire : "Sa table était frugale, mais tout autour de la salle à manger régnaient les bois des cerfs tués par ses aïeux ». Le lecteur comprend aisément que ce père n'avait «pas beaucoup de mérites » autres que l'ancienneté de sa noblesse, directement liée à la chasse, à la richesse et à l'abondance de ses repas :

Il se rappelait, les jours gras, qu'il avait droit de chasse, les jours maigres, qu'il avait droit de pêche, et content de ces droits, il laissait sans envie manger des faisans et des carpes aux ignobles financiers.

Il chassait quelquefois, et mangeait son gibier avec les filles du cabaret voisin ; il buvait beaucoup et jouait tous les soirs avec son domestique. Sa figure était désagréable, $[\ldots]^{30}$.

On devine que le baron, qui est veuf, constitue une catastrophe potentielle par rapport au bonheur futur de sa fille. Celle-ci aura en effet le tort de vouloir épouser un jeune homme riche, certes, et de famille noble - mais de noblesse trop récente pour qu'il soit acceptable aux yeux du baron $^{31}$. La fille sera donc enfermée dans une tour du château. Le lecteur s'y attendait... Mais Julie réussira à se libérer - on s'y attendait également.

La nourriture joue un beau rôle pour préparer l'évasion de Julie : la romancière y fait appel en créant pour ainsi dire une sous-catégorie du topos narratif RUSER_PAR_AMOUR. La ruse est possible grâce au transport de la nourriture ${ }^{32}$ : le baron faisant garder sa fille par

\footnotetext{
${ }^{30} O C$, t. VIII, p. 21

31 Le cas pourrait se formuler comme topos narratif: *ECART_CLASSE_EPOUX (selon le modèle de *ECART_AGE_AMOUREUX/EPOUX. Voir Suzan van Dijk, « Topoi et généricité », 2002, p. 445), avec cette précision que le père de Julie s'y oppose, et qu'il s'agit donc, aussi, du topos CONDITION_EMPECHER_MARIAGE (Ibid., p. 451).

${ }^{32}$ Voir l'Index des topoi contenu dans Elzbieta Grodek, Ecriture de la ruse, 2000, p. 451. Il faudrait cependant que la phrase correspondant à cette dénomination ait une portée plus large que celle que l'on trouve p. 393 : « Un personnage emploie la ruse pour que la personne qu'il aime lui rende son amour » (mes italiques). Faut-il proposer *NOURRITURE_AIDER_FUITE ? Ce serait à vérifier dans d'autres romans. En fait c'est surtout grâce à la jeune fille, à son bon caractère et à la solidarité entre les deux femmes que les choses se passent si bien, ce qui semble
} 
deux de ses servantes, la plus jeune des deux, qui a pour tâche d'apporter à manger à Julie, en profite pour également faire passer des messages entre Julie et son amant. C'est ainsi que Julie réussit à s'évader et à retrouver Valaincourt. Le père se console grâce à son fils : celui-ci lui amène une bru qui, bien que « louche et bossue $»^{33}$, est « complètement noble » : « le mariage [entre eux] fut donc bientôt résolu $»^{34}$.

C'est ce qui nous mène évidemment à un repas de noces organisé pour le fils. Le lecteur n'est pas renseigné sur le menu, mais il apprend qu'à la fin du dîner le père « célébra par vingt rasades une union si bien assortie ». Julie, connaissant bien son père, avait compté là-dessus et choisi son moment : «Lorsque le vin commençait à confondre dans sa tête l'ancienne et la nouvelle noblesse, Valaincourt et Julie entrèrent dans la salle et se jetèrent à ses pieds : ayant perdu une partie de ce qu'il appelait sa raison, il ne sentit que sa tendresse, et pardonna. Julie fut heureuse, et ses fils ne furent point chevaliers $»^{35}$. Tout le monde est content, et il semble bien que nous voyions là le topos du DENOUEMENT_MARIAGE ${ }^{36}$ ! Ce n'est pas un petit dîner intime, mais un événement festif réunissant de nombreux convives, de telle sorte que le pardon du baron vis-à-vis de Julie est assuré d'un public qui peut voir, applaudir - ou s'indigner peut-être - mais en tout cas, témoigner.

Ce premier ouvrage de la très jeune Belle montre bien qu'elle réussit à utiliser, de façon très convaincante, des éléments liés aux habitudes alimentaires pour (1) caractériser ses personnages (en l'occurrence le père de Julie : non seulement chasseur, mais abusant de l'alcool), et pour (2) amener des scènes où des individus peuvent être opposés à un groupe qui, soit fait d'autant mieux ressortir des individualités, soit constitue un public (comme lors du dîner au dénouement) - la présence du public permettant la légitimité de cet accord qui en tant que tel n'était pas légitime, puisque donné sous l'effet de l'alcool.

\section{Lettres neuchâteloises (1784)}

Dans son second roman, publié plus de dix ans après son émigration, les Lettres neuchâteloises, on suit Henri Meyer, jeune Allemand venu travailler dans un bureau de commerce, et qui s'intègre de plus en plus dans la ville de Neuchâtel. Cette intégration se

aussi amener le topos FEMME_AIDER_FEMME. Voir mon article « Amitié, solidarité et entraide féminines : Spécificités d'auteurs femmes?», Topiques, Études Satoriennes, 2015.

${ }_{33}$ Serait-ce un renversement du topos SEDUIRE_PAR_CHARMES? Voir Suzan van Dijk, «Topoi et généricité », 2002, p. 447.

${ }_{34}^{34}$ Le Noble in $O C$, t. VIII, p. 34.

${ }^{35}$ Idem. On pourrait imaginer ici l'utilité d'un topos comme: *ALCOOL_FACILITER_SOLUTION ou CONTACT ?

${ }^{36}$ Voir Suzan van Dijk, « Topoi et généricité », op. cit., p. 467. 
constate notamment au fur et à mesure de sa présence à des repas, dont il parle dans des lettres envoyées à un ami. Au début du roman il avait déclaré ne pas trop aimer ce genre de visites, pour lesquelles on dépend d'une invitation et où, une fois invité, on n'est pas sûr de se sentir à l'aise. Dans sa deuxième lettre il décrivait (à ce même ami) sa nouvelle vie, et les contacts avec ses collègues, ainsi qu'avec les cercles un peu plus élevés - contacts qui commencent à se créer justement autour de la nécessité de se nourrir :

[...] les apprentis et les commis mes camarades ne me plaisent ni ne me déplaisent: nous mangeons tous ensemble, excepté quand on m'invite chez mon patron, ce qui est arrivé deux fois en quatre jours : tu vois que cela est fort honnête ; mais je m'y amuse aussi peu que je m'y ennuie ${ }^{37}$.

Il y a, évidemment, une distinction entre le niveau des employés de ce bureau et celui du patron; Henri est invité à passer d'un niveau à l'autre, mais il a tendance à refuser. Quelques mois plus tard, dans la lettre 9, il exprime encore les mêmes sentiments d'ennui, et semblerait confirmer sa position d'étranger : il formule un certain agacement à propos de ce type de dîners conçus pour impressionner, et qui ne conviendraient pas aux caractères plutôt timides comme le sien :

Je me suis bien ennuyé aujourd'hui, mon cher ami. Mon patron a eu la bonté de me faire inviter à un grand dîner, où l'on a plus mangé que je n'ai vu manger de ma vie, où l'on a goûté et bu de vingt sortes de vins. Bien des gens se sont à-demi grisés, et n'en étaient pas plus gais ${ }^{38}$.

Mais, ayant fait la connaissance d'une certaine Mlle de La Prise, qui l'intéresse pour plusieurs raisons, il commence à se sentir intégré dans la société neuchâteloise. L'établissement du contact est décrit dans la lettre 19, qui commence par : «Hier, après dîner, le comte Max vint me prendre pour me mener promener ». Les deux hommes vont faire une promenade de début d'après-midi et rencontrent cette même Marianne de La Prise se promenant avec une cousine. Comme il commence à pleuvoir, elle invite les deux hommes à la maison. La famille est française, son père Officier retiré du service, visiblement très attaché à sa fille. Alors les deux jeunes hommes se voient offrir non pas un « grand dîner », mais un goûter sympathique, improvisé, dont - pour une fois - la composition est communiquée au lecteur :

On nous a donné du thé, des raisins, de petits gâteaux. Ce petit repas, qui jusqu'ici m'avait paru assez mal entendu, m'a paru hier fort agréable. Je croyais être en famille avec M. de la Prise et Mlle Marianne. Elle ne m'offrait rien que je n'acceptasse. Elle choisissait des grappes pour le comte Max et pour moi. Pour la première fois je n'étais plus un étranger à Neuchâte $\mathrm{l}^{39}$.

\footnotetext{
${ }^{37} O C$, t. VIII, p. 49.

${ }^{38} O C$, t. VIII, p. 57. Il semble se trouver ici une occurrence d'un possible topos dépréciant l'abus de l'alcool.

${ }^{39} O C$, t. VIII, p. 75.
} 
Sur l'invitation du père, qui brusque légèrement sa femme, Henri et son ami resteront même à souper pour un repas à nouveau très simple - comme l'indique bien cette précision : ce sera une omelette. Henri mange alors, certes, mais il profite surtout de l'occasion de voir et notamment d'observer Marianne, entourée de sa famille, et de tirer des conclusions :

[...] Le souper était propre et simple. Il faut avouer que Mme de la Prise n'en faisait pas trop maussadement les honneurs. Sa fille était très gaie : son père paraissait enchanté d'elle ; et sûrement ses convives ne l'étaient pas moins ${ }^{40}$.

On note ici l'effet sur l'observateur de cette « éloquence du corps » dont traite Monique Moser-Verrey pour la même scène ${ }^{41}$ : le regard de l'autre en est le complément nécessaire. Tout est non seulement $v u$, mais aussi écrit par Meyer dans cette lettre à son ami, notamment la bonne humeur du père qui pousse sa femme à aller au-delà des gâteaux et des raisins.

Dans ce roman Henri Meyer n'est pas le seul à désirer - du moins dans un premier temps - se soustraire aux repas pris en commun : un personnage que les autres trouvent un peu bizarre, et qu'ils appellent « le caustique », semble aussi (d'après la lettre 18) préférer s'éloigner de ces compagnies de mangeurs et de buveurs. Il s'en explique à la fin d'un dîner chez le patron :

On s'était levé de table et on prenait du café, quand le Monsieur caustique du bal est entré. On lui a reproché de n'être pas venu plus tôt.

- Je vous suis obligé, a-t-il répondu, mais je ne mange presque jamais hors de chez moi, depuis que je connais parfaitement les vins de tous vos quartiers et le fromage de toutes vos montagnes ${ }^{42}$.

Sa raison pour participer, autrefois, à des repas hors de chez lui n'avait pas tenu à la gourmandise ni au plaisir de la convivialité autour d'une table : il avait juste voulu connaître la production locale en vins et en fromages. Étant suffisamment informé, il n'y avait plus de raison pour lui de participer à ces repas. C'est un personnage plus durablement solitaire qu'Henri, auquel on ne peut, tout au moins, pas faire le reproche d'être un gros mangeur.

\section{Lettres de Mistriss Henley (1784)}

Dans les Lettres de Mistriss Henley, parues la même année, Charrière revient - par un détail qui est significatif - sur la question des gros mangeurs dont il s'agit de se méfier. Lorsque Mistriss Henley explique à son amie comment elle avait choisi son mari entre deux prétendants qu'elle avait eus étant jeune, voici comment elle les oppose :

\footnotetext{
${ }^{40}$ Ibid., p. 76

${ }^{41}$ Monique Moser-Verrey, op. cit., p. 127.

${ }^{42}$ OC, $t$. VIII, p. 72.
} 
[le premier] était un bel homme; il était noble dans ses manières et dans sa dépense; il aimait la bonne chère, les arts et les plaisirs: je lui plus [...]. L'autre homme à marier était le second fils du comte de Reding, âgé de trente-cinq ans, veuf $[\ldots]$. Je trouvai que tout ce qu'il disait répondait à cet extérieur si agréable. Il m'entretint souvent de la vie qu'il menait à la campagne, [...].

Et c'est ce dernier, M. Henley, qu'elle choisit, car « me donner moi-même de mon choix, contre des diamants, des perles, des tapis, des parfums, des mousselines brodées d'or, des soupers, des fêtes, je ne pouvais m'y résoudre $»^{43}$.

Cependant, comme elle s'en rend compte maintenant, Mrs Henley avait fait une erreur : la sobriété d'un homme ne signifie pas forcément qu'il faille le choisir comme époux. M. Henley a beau être sobre, c'est aussi un homme très raisonnable, qui a toujours raison et tient à avoir raison. Mrs Henley était devenue fort malheureuse dans ce mariage, au point que dans la dernière de ces cinq lettres, elle suggère que sa propre mort - soit spontanée soit suicide pourrait être prochaine ${ }^{44}$.

\section{Trois femmes (1795)}

Il n'en reste pas moins que la préférence de l'auteure (chez elle, et chez les personnages qu'elle entend mettre en valeur) pour une vie relativement simple, avec des repas correspondants, semble bien être une constante. Dans Trois femmes, qui date d'après la Révolution, cette tendance est même accentuée : on y voit comment une émigrée française, une orpheline appelée Emilie, partie en Allemagne avec sa servante Joséphine, apprend à vivre « simplement » et plus près de la nature, grâce notamment aux « leçons » de cette servante. Elles ont trouvé une petite maison où se loger, et il va être question d'un jardin potager dont Joséphine sera responsable :

Joséphine cultivait toutes sortes de légumes, nourrissait une chèvre, filait du chanvre et du lin. Emilie arrosait quelques rosiers, caressait la chèvre, brodait de la mousseline et du linon, dont Joséphine était parée le dimanche et les jours de fête. On vivait simplement et sainement ${ }^{45}$.

La simplicité (ou sobriété, voire frugalité) - pas seulement en nourriture d'ailleurs devient la règle, même si Emilie ne quitte pas tout à fait son rôle, et continue de faire de la broderie et d'aimer jouer de la harpe. Lorsqu'Emilie et Joséphine rencontrent la troisième des

\footnotetext{
${ }^{43} O C$, t. VIII, p. 103-4.

${ }^{44}$ Voir à propos du mariage que décrit Charrière dans ce roman, Martine Reid, «Dilemme, ou la condition des femmes », 2006.

${ }^{45} O C$, t. IX, p. 44.
} 
«trois femmes », Constance, qui elle-même est riche ${ }^{46}$, Emilie propose de se lier d'amitié - ce qui est possible justement parce que, malgré sa richesse ${ }^{47}$, Constance ne semble pas faire partie des « gros mangeurs » :

[...] revoyons-nous demain et tous les jours et à toute heure. Vous êtes riche, à ce que je vois, et je suis pauvre; mais comme vous ne me paraissez ni fastueuse ni sensuelle, nous n'en pourrons pas moins vivre ensemble, et je consens à ne pas compter trop juste avec une amie ${ }^{48}$.

Le message central du roman Trois femmes est formulé d'abord à l'aide justement de ces renvois à la nourriture de luxe et autres démonstrations de richesse, qu'il faudrait essayer de réduire. C'est Constance qui l'exprime dans une des lettres qu'elle adresse - à propos d'une lecture qu'elle venait de faire - au personnage narrateur, l'abbé de La Tour :

Quelqu'un disait à un nouveau riche : Vous soupez bien et donnez souvent à souper à vos amis : c'est fort bien fait, mais par égards pour vos voisins, mettez une sourdine à votre tournebroche. [...] j'espère que partout on va épargner le bruit du tournebroche à celui qui ne devra pas manger du rôti. J'espère que partout chacun voilera son luxe; la prudence le veut.

Pour être complètement claire, l'auteure élimine ensuite la terminologie directement liée à la nourriture :

La générosité exige davantage, elle veut qu'on diminue le luxe privé, les jouissances égoïstes, et que les grandes fortunes se popularisent. Riches, si vous voulez qu'on vous pardonne vos richesses, ne vous contentez pas d'être charitables : soyez généreux ${ }^{49}$.

La nourriture est donc mise au service du discours égalitaire, dans lequel la générosité notamment celle des riches et des dirigeants - est un concept qui apparaît régulièrement ${ }^{50}$.

\section{Sainte Anne (1799)}

Dans un des derniers romans d'Isabelle de Charrière, Sainte Anne, on retrouve à nouveau la structuration de la journée rendue possible par le repas en commun, avec des formules comme : «D'abord après-dîné, il alla chercher Mademoiselle d'Estival ${ }^{51} »$. Cette Mademoiselle d'Estival est, à côté du jeune Ste. Anne, le personnage central du roman : fille illégitime d'un noble de la région et d'une de ses domestiques, le père lui avait donné son nom,

\footnotetext{
${ }^{46}$ D'une richesse d'ailleurs suspecte, elle s'en rend compte.

${ }^{47}$ Ou plutôt : parce qu'elle tient à compenser l'origine douteuse de sa richesse.

${ }^{48} O C$, t. IX, p.63.

${ }^{49} O C$, t. IX, p. 109.

${ }^{50}$ Voir par exemple, à ce propos sa brochure intitulée Réflexions sur la générosité et sur les princes, intégrée dans ses Observations et conjectures politiques en 1787 (OC X, p. 70-73).

${ }^{51} O C$, t. IX, p. 302.
} 
mais n'avait pas fait en sorte qu'elle sache lire ${ }^{52}$. Par contre, grâce à sa mère, elle se connaît parfaitement en herbes - mauvaises aussi bien que médicinales. Pour Ste. Anne, qui est en train de tomber amoureux d'elle, c'est là que réside une expertise importante, et par conséquent une certaine autorité de guérisseuse, annulant l'inégalité qui existe officiellement entre eux.

Elle est entourée d'autres jeunes-filles - notamment des riches, légitimes, sachant lire qui s'intéressent à Ste. Anne. Ne reconnaissant pas comme telle l'expertise de Mlle d'Estival, les autres s'amusent de son analphabétisme. Plusieurs fois tous vont se retrouver autour d'une table à manger. Les regards alors se croisent par-dessus les mets que l'on sert. Ce qui importe, ce n'est pas la nourriture, c'est la confrontation entre ces jeunes femmes dont une, Mlle de Rhedon, non seulement se destinerait bien à épouser Ste. Anne, mais est aussi celle que Mme Ste. Anne (qui est veuve) préfère pour son fils. D'où une certaine tension notamment entre les jeunes filles de bonne famille par rapport à Mlle d'Estival, qu'elles croient superstitieuse, et qu'elles essayent - en vain - d'éliminer :

À l'heure du dîner ils rentrèrent. « Je ne pense pas que Mlle d'Estival veuille dîner avec nous », dit Mlle de Kerber, « nous serions treize à table ». Mlle d'Estival compta attentivement. "Non», dit-elle, «nous ne serons que onze, et elle s'assit $»^{53}$.

Pendant une scène cruciale, les deux « rivales », Mlles de Rhedon et d'Estival, se trouvent à table à nouveau, côte à côte et entourées des autres jeunes filles, Ste. Anne étant absent. On apporte alors à Mlle d'Estival une lettre, que lui envoie un ami de Ste. Anne - lettre qui, par définition, ne lui sert pas à grand-chose. C'est à propos de cette lettre qu'on voit - et toutes autour de la table en sont témoins - que la rivalité entre les deux filles peut ne pas exclure la solidarité :

« J'ai toute confiance en vous », ajouta Mademoiselle d'Estival, s'adressant toujours à Mademoiselle de Rhedon, « voudriez-vous prendre la lettre et la lire [...] ?». Mademoiselle de Rhedon fut extrêmement troublée, mais elle dissimula son trouble. Mademoiselle d'Estival la pria de lui dire ce qu'elle avait lu. " Non pas dans ce moment », lui dit son amie, d'une voix extrêmement changée $[\ldots]^{54}$.

La rivale amie avait constaté qu'au fond la lettre la concerne tout autant que Mlle d'Estival : elle y apprend que Ste. Anne aime Mlle d'Estival et espère l'épouser. C'est ce qui va en effet arriver : il y aura ici à nouveau un DENOUEMENT_MARIAGE. Mais autour de ce repas (dont le menu reste inconnu) les autres personnes participent aux émotions : elles voient

\footnotetext{
${ }^{52}$ Comme Isabelle de Charrière l'écrit à un ami genevois : « Mon dernier roman commence par : "Elle ne sait pas lire", et c'est de l'héroïne que cela se dit » (lettre 1814 à Marc-Auguste Pictet, 31 juillet 1797, OC, t. V, p. 336).

${ }^{53} O C$, t. IX, p. 268.

${ }^{54} O C$, t. IX, p. 304.
} 
comment amitié et solidarité se manifestent ${ }^{55}$, au point même qu'il y aura - comme dans $L e$ Noble - un double mariage : il s'avère qu'un ami de Ste. Anne était amoureux de Mlle de Rhedon.

\section{Conclusion : la romancière mentor de son public?}

Il semble bien clair que dans ces romans nous retrouvons des préoccupations propres à Isabelle de Charrière, telles que formulées dans ses lettres - préoccupations qui ne concernent pas vraiment la nourriture elle-même. En tant que romancière elle ne fait que se servir du « boire et manger » pour donner une forme narrative à ses idées : mettant face à face des personnages qui non seulement sont de conditions sociales différentes, mais se situent aussi de façon différente par rapport à la convivialité des repas. Certain-e-s ont tendance à se poser comme mentor ou conseillère, grâce à une certaine autorité qui peut être reconnue, ou non, par les autres. Les topoi que l'on pourrait reconnaître ici, et qui sont donc liés à des notions de ruse, intégration, et, surtout, de mentorat, profitent en quelque sorte, de certains éléments liés au «boire et manger », mais ils pourraient tout aussi bien se combiner avec d'autres activités humaines qui seraient pratiquées en commun.

De la même façon que dans ses lettres l'épistolière manifestait volontiers, à propos de nourriture et de repas à prendre, son naturel de mentor, on retrouve, dans les scènes que nous venons de passer en revue, le topos du MENTORAT, comme une constante qui fournirait le cadre. Les personnages attablés préfigurent peut-être aussi, à l'intérieur du roman, le public des lecteurs qu'il s'agit de convaincre - et à qui elle adresse le message « démocratique » qu'elle tient à distribuer, comme elle l'avait explicitement formulé dans sa lettre à son traducteur allemand Ludwig Ferdinand Huber ${ }^{56}$.

Ainsi le rapport entre lettres et romans concerne les deux directions : les lettres n'étant pas seulement un métadiscours entourant le roman, mais les romans étant aussi le « prolongement» de ce qu'elle dit dans ses lettres. Ce qui fait comprendre qu'elle ait pu écrire à son neveu, qui avait envie de connaître ses ouvrages : « N'ayez donc, pour l'heure, aucun regret à mes ouvrages. Ils ne sont d'ailleurs pas différents de mes lettres ». Et elle précise : « [...] je dis aussi là, comme ici, ce que je pense sur des objets qui me paraissent intéressants. Mes lettres sont donc de tout petits livres en manuscrit $»^{57}$.

\footnotetext{
${ }^{55}$ Situation à laquelle on peut appliquer le topos FEMME_AIDER_FEMME ; voir Van Dijk, « Amitié, solidarité et entraide féminines : Spécificités d'auteurs femmes ?», 2015.

${ }^{56}$ Citée plus haut, voir n. 28.

${ }^{57}$ Lettre 1911 à son neveu Willem-René de Tuyll, le 4 avril 1798, OC, t.V, p. 429.
} 


\section{BIBLIOGRAPHIE}

\section{Euvres et sources}

CHARRIERE, Isabelle de, Euvres complètes d'Isabelle de Charrière / Belle de Zuylen, Amsterdam, van Oorschot, 1979-1984, 10 vols. (éds. Jean-Daniel CANDAUX, C.P. COURTNEY, Pierre H. DUBOIS, Simone DUBOIS-DE BRUYN, Pierre THOMPSON, Jeroom VERCRUYSSE ET Dennis M. WOOD).

\section{Études}

Belle de Zuylen / Isabelle de Charrière. Education, création, réception, VAN DIJK, Suzan, COSSY, Valérie, MOSER-VERREY, Monique, VAN STRIEN-CHARDONNEAU, Madeleine (eds.), Amsterdam / New York, Rodopi (Coll. Faux-Titre, 276), 2006.

CITTON, Yves, «Les spectres de la multiplicité. La littérature du XVIII ${ }^{\mathrm{e}}$ siècle revisitée depuis ses dehors », dans Christie McDONALD et Susan Rubin SULEIMAN (eds.), French Global. Une nouvelle perspective sur l'histoire littéraire, Paris, Classiques Garnier, 2014, p. 551-576, en ligne : https://hal.archives-ouvertes.fr/hal-01373195/document [cons. 2-32021]).

Écriture de la ruse, Elzbieta GRODEK, (ed.), Amsterdam / Atlanta, Rodopi (Coll. Faux-Titre, 190), 2000.

JONGBLOED, Hein H. et VAN DIJK, Suzan, « De vier "nieuwe" brieven van Belle van Zuylen », Tirade, 2012, n 56, p. 91-99.

KLOEK, Joost et MIJNHARDT, Wijnand, Dutch Culture in a European Perspective 2; 1800; Blueprints for a National Community, Londres, Palgrave Macmillan, 2004.

MOSER-VERREY, Monique, Isabelle de Charrière, salonnière virtuelle: Un itinéraire d'écriture au XVIII' siècle, Paris, Hermann, 2013.

NOORDERVLIET, Nelleke, Volg je eigen weg, postface à Belle VAN ZUYLEN, De Edelman (trad. du Noble par Rosalien VAN WITSEN), Amsterdam, Van Oorschot, 2013, p. 3549.

PELCKMANS, Paul, «Adieu Poes que je chéris, quoiqu'il me mette de mauvaise humeur.... Isabelle de Charrière et Willem-René van Tuyll », dans VAN DIJK et al., Belle de Zuylen 
/ Isabelle de Charrière. Education, création, réception. Amsterdam / New-York, Rodopi (Coll. Faux-Titre, 276), 2006, p. 69-84.

REID, Martine, «Dilemme, ou la condition des femmes », dans VAN DIJK et al., Belle de Zuylen / Isabelle de Charrière. Education, création, réception, Amsterdam / New York, Rodopi, (Coll. Faux-Titre, 276), 2006, p. 167-174.

VAN DEN BERGH, Greetje, Ik heb geen talent voor ondergeschiktheid. Belle van Zuylen in briefwisseling met Constant d'Hermenches, James Boswell en Werner C.W. van Pallandt. Amsterdam, Van Oorschot, 1987.

VAN DIJK, Suzan, « Topoi et généricité : inventaire et classement des topoi présentés », dans Suzan VAN DIJK et al., (éds.), Féminités et masculinités dans le texte narratif avant 1800. La question du 'gender', Louvain, Peeters (Coll. La République des Lettres, 6), 2002, p. 443-467.

VAN DIJK, Suzan, « Belle de Zuylen et les 'talents' des Hollandaises », in Cahiers Isabelle de Charrière / Belle de Zuylen Papers, 2010, $\mathrm{n}^{\circ}$ 5, p. 64-74.

VAN DIJK, Suzan et SCHOUTEN, Maria, « Numériser les lettres de Belle de Zuylen: Un regard plus précis sur les rapports familiaux », in Cahiers Isabelle de Charrière/Belle de Zuylen Papers, 2013, nº 8, p. 24-44.

VAN DIJK, Suzan, «Amitié, solidarité et entraide féminines: Spécificités d'auteurs femmes ? », Topiques. Études Satoriennes, éd. Hélène Cazes, 2015/1,

http://journals.uvic.ca/index.php/sator/article/view/12633.

VAN DIJK, Suzan, en collaboration avec SCHOUTEN, Maria, « La correspondance d'Isabelle de Charrière (1740-1805). Survie et destruction des lettres comme thématiques de l'épistolière », dans Matthieu MAGNE (éd.), Prendre la plume des Lumières au Romantisme, Clermont-Ferrand, Presses Universitaires Blaise Pascal, 2019, p. 149-164.

VAN DIJK, Suzan, « Belle van Zuylen: schrijfster van adel, over de adel. Haar correspondentie digitaal beschikbaar », à paraître dans Virtus, Jaarboek voor Adelsgeschiedenis. 2021.

VAN STRIEN-CHARDONNEAU, Madeleine, " Isabelle de Charrière, pédagogue », dans VAN DIJK et al., 2006, Suzan VAN DIJK et al., Belle de Zuylen / Isabelle de Charrière. Education, création, réception, Amsterdam / New York, Rodopi (Coll. Faux-Titre, 276), 2006, p. 49-68.

VISSIERE, Isabelle et VISSIERE, Jean-Louis (éds.), Isabelle de Charrière. Une liaison dangereuse: Correspondance d'Isabelle de Charrière avec Constant d'Hermenches, 1760-1776, Paris, La Différence, 1991. 
WENT-DAOUST, Yvette, «La place des Lettres neuchâteloises dans le roman épistolaire du XVIIIe siècle », in Doris JAKUBEC et Jean-Daniel Candaux (éds)., Une Européenne : Isabelle de Charrière en son siècle, Neuchâtel, Eds. Gilles Attinger, 1994, p. 187-196. 